\title{
Actions et missions actuelles de la Société Française de Physique
}

En ce mois de février, s'il est un peu trop tard pour présenter ses vœux, il est toujours intéressant de revenir sur l'année passée et d'énoncer les « bonnes résolutions » prises pour 2019.

En 2018, grâce à l'engagement de toute notre communauté, la Société Française de Physique s'est investie dans divers débats et a organisé de nombreux évènements dans toute la France, dont vous pourrez bientôt retrouver les détails dans notre rapport d'activités.

Nous pouvons tout particulièrement rappeler les Journées de la Matière Condensée ${ }^{(*)}$, organisées à Grenoble, qui ont permis à environ 700 participants d'échanger sur les sujets émergents de leur discipline ainsi que sur des thèmes de « science et société ». Citons aussi les Rencontres de Jeunes Physiciens qui ont rassemblé à Paris et Grenoble des doctorants et des postdoctorants, en leur donnant l'opportunité de pratiquer leur expression orale et de découvrir la richesse des réseaux et de l'interdisciplinarité. Enfin, la conférence sur l'enseignement des sciences du 10 février 2018, suite au courrier adressé au ministre Jean-Michel Blanquer (conjointement avec la SCF, I'UdPPC et I'UPS $\left.{ }^{(*)}\right)$ a permis d'exprimer notre position sur plusieurs points relatifs à la formation scientifique, et plus particulièrement en physique-chimie.

Sous l'impulsion de notre vice-président sortant, Michel Spiro, nous avons travaillé au rapprochement de la SFP avec les sociétés savantes sœurs. Ce rapprochement s'est traduit notamment par une prise de position commune avec certaines d'entre elles en faveur du Palais de la découverte, par la rédaction d'un « cahier d'acteur » avec la Société Chimique de France pour contribuer au débat public sur la programmation pluriannuelle de l'énergie, ou encore par l'organisation d'évènements communs dont la 4 e Journée Sciences \& Médias consacrée à la lutte contre la désinformation scientifique.

Enfin, citons l'entrée du Rayon (site de la Commission Jeunes ayant pour vocation de permettre à de jeunes chercheuses et chercheurs de publier des articles de vulgarisation autour de leurs sujets de recherche) au Café des Sciences, association réunissant un grand nombre d'acteurs et d'actrices du monde de la vulgarisation scientifique, dans des domaines très variés dont la physique bien sûr.

L'année 2019 promet d'être riche en évènements majeurs avec, notamment, la tenue du Congrès Général à Nantes en juillet, mais aussi la Nuit de l'antimatière en avril, ou encore la finale des Olympiades de Physique et le French Physicist's Tournament en février pour n'en citer que quelques-uns.

La SFP aura 150 ans en 2023. C'est une belle occasion de se projeter dans le futur. Le séminaire annuel de décembre dernier rassemblant des représentants de nos composantes (bureau, conseil d'administration, divisions, commissions et sections locales) a permis d'y travailler, de confirmer nos valeurs et de décliner les initiales de la SFP pour réaffirmer nos missions :

Stimuler le développement des connaissances en physique,

Fédérer la communauté des physiciennes et physiciens,

Promouvoir et faire rayonner la physique.

Plusieurs thèmes sont ressortis pour alimenter la vision de l'avenir que nous souhaitons donner collectivement à la SFP. Deux sont apparus comme prioritaires : une ouverture plus grande vers les jeunes, et le renforcement de notre ambition citoyenne pour faire entendre la voix de

${ }^{(*)}$ Voir l'éditoria du numéro 59 de Reflets de la physique (octobre 2018)

${ }^{(* *)}$ Société Chimique de France, Union des Professeurs

de Physique

et de Chimie

et Union des

Professeurs

de classes

préparatoires

Scientifiques la communauté auprès des pouvoirs publics et de la société. La SFP réaffirme aujourd'hui sa volonté d'apporter au débat public son expertise et un éclairage scientifique indépendant, que ce soit dans le domaine de l'enseignement de la physique pour former la nouvelle génération aux défis qu'elle aura à relever, ou dans des domaines spécifiques tels que celui de l'énergie. Le premier débat organisé le 19 janvier de cette année et consacré au thème « climat, énergie, environnement » en est une belle illustration.

L'ambition est clairement affichée et nous vous informerons régulièrement des résultats obtenus grâce à l'engagement de chacune et chacun d'entre vous. Merci à toutes et à tous pour vos contributions! 